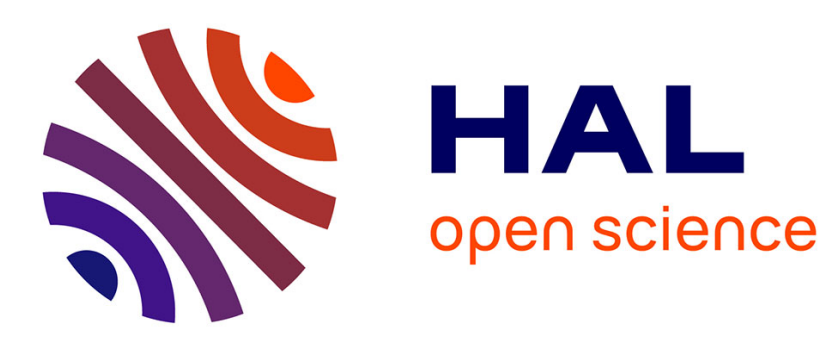

\title{
Identification et analyse des prises de décision dans la chaîne de soins en radiothérapie
} Alain Genzling, Bruno Chauvin, Odile Rohmer

\section{To cite this version:}

Alain Genzling, Bruno Chauvin, Odile Rohmer. Identification et analyse des prises de décision dans la chaîne de soins en radiothérapie. Psycho-Oncologie, 2016, 10 (3), pp.201-210. 10.1007/s11839-0160587-x . hal-02167609

\section{HAL Id: hal-02167609 https://hal.science/hal-02167609}

Submitted on 27 Jun 2019

HAL is a multi-disciplinary open access archive for the deposit and dissemination of scientific research documents, whether they are published or not. The documents may come from teaching and research institutions in France or abroad, or from public or private research centers.
L'archive ouverte pluridisciplinaire HAL, est destinée au dépôt et à la diffusion de documents scientifiques de niveau recherche, publiés ou non, émanant des établissements d'enseignement et de recherche français ou étrangers, des laboratoires publics ou privés. 
Gentzling, A., Chauvin, B., \& Rohmer, O. (2016). Identification et analyse des prises de décision dans la chaîne de soins en radiothérapie. Psycho-Oncologie, 10, 201-210. doi: 10.1007/s11839-016-0587-x

\title{
Identification et analyse des prises de décision dans la chaîne de soins en radiothérapie
}

\author{
A. Genzling - B. Chauvin - O. Rohmer
}

Laboratoire de Psychologie des Cognitions, EA 4440

Université de Strasbourg, 12 rue Goethe, F-67000 Strasbourg

Toute correspondance au sujet de cet article peut être adressée à Bruno Chauvin (bruno.chauvin@unistra.fr) 
Résumé Le double objectif de cette recherche a été de mettre en évidence l'impact des interactions sociales dans les prises de décisions en radiothérapie et d'identifier en quoi ces décisions peuvent être liées à des risques potentiels pour le patient. Pour ce faire, nous avons analysé l'activité réelle de préparation des traitements des patients au sein d'un service de radiothérapie. Les résultats de notre analyse ont fait apparaître huit situations dans lesquelles des décisions doivent être prises, dont la moitié sont sous l'influence des interactions entre professionnels et avec les patients, et qui présentent toutes des risques potentiels pour la santé de ces derniers. Cette analyse de terrain met donc en évidence la nécessité de prendre en compte les échanges sociaux informels dans l'identification des risques inhérents aux soins en radiothérapie et propose une analyse plus spécifique des mécanismes psychosociaux en jeu.

Mots clés Prise de décision - Interactions sociales - Risque - Radiothérapie - Analyse de l'activité 
Abstract The purpose of this study was twofold: first, we wanted to understand what role social interactions play in decision making processes during radiotherapy; second, we aimed to highlight in what extent these decisions can create patient risk. Our analysis focused on the real existing activity which is performed for preparing radiotherapy treatments. We found eight situations in which decision making is needed: for half of them, interactions among professionals or between professionals and patients have a key role; for all of them, decision making implies potential risks for patients. These findings stress the importance of taking into account social informal interactions to better understand decision making and its inherent risks for patients treated with radiotherapy. To conclude, some psychosocial processes that could be involved in these situations are discussed.

Keywords Decision making - Social interactions - Risk - Radiotherapy - Analysis of the real activity 


\section{Introduction}

Cette recherche s'inscrit dans une démarche d'amélioration de la sécurité des soins en radiothérapie qui prend en considération la dimension psychosociale au même titre que les facteurs techniques et organisationnels.

La radiothérapie est l'un des trois traitements majeurs du cancer, aux côtés de la chirurgie et de la chimiothérapie. C'est un traitement très efficace puisqu'on estime aujourd'hui que près de la moitié des cancers guéris le sont grâce à la radiothérapie, seule ou associée à d'autres traitements [1]. D'un point de vue technique, la radiothérapie consiste à utiliser des rayonnements ionisants (rayons X pour l'essentiel) pour détruire les cellules cancéreuses. Ces rayonnements sont produits par des accélérateurs de particules pouvant tourner sur $360^{\circ}$ autour du patient allongé sur une table de traitement. La radiothérapie peut être utilisée soit à visée curative, pour guérir, soit à visée palliative, pour soulager quand il n’y a plus d'espoir de guérison, soit à visée symptomatique, pour supprimer un symptôme [1].

Depuis les années 1970, la spécialité de radiothérapie a continuellement évolué sur les plans technique et technologique, permettant la réduction des risques associés à ce type de traitement [2]. L'amélioration des techniques d'imagerie a considérablement réduit le risque d'irradier des organes sains ; l'accumulation des connaissances médicales sur l'effet des doses de rayons délivrées a permis de mieux maîtriser le risque d'apparition d'effets secondaires ; l'évolution des techniques de traitement, de plus en plus précises, a contribué à améliorer la sécurité des soins dispensés aux patients [3]. Encore aujourd'hui, la démarche d'amélioration de la gestion des risques en radiothérapie se focalise sur la dimension technique de cet environnement de travail.

Cependant, comme en témoigne notamment l'accident majeur qui a touché le service de radiothérapie de l'hôpital d'Epinal en 2004-2005, ces évolutions techniques n'ont pas été suffisantes pour permettre la pleine maîtrise des risques présents dans cet environnement, 
soulignant l'importance de facteurs d'une autre nature dans la réalisation de tels risques.

L'analyse de cet accident - qui a causé la sur-irradiation de plusieurs milliers de patients et le décès de plusieurs d'entre eux - a en effet montré que celui-ci est la résultante d'une succession de défaillances organisationnelles (ex. manque de formation du personnel) et humaines (ex. non-prise en compte d'une information de contrôle) [4,5], soulignant ainsi l'importance de ces deux facteurs dans la survenue d'un tel accident.

Pour remédier à ces défaillances, un nouveau cadre règlementaire pour la radiothérapie fixant des obligations de sécurité établies par deux organismes indépendants - la Haute Autorité de Santé (HAS) et l'Autorité de Sûreté Nucléaire (ASN) - a été élaboré. Aujourd'hui, pour être certifiés, les établissements de santé doivent remplir un certain nombre d'obligations de sécurité et de qualité des soins fixées par la HAS [6]. Concernant les services de radiothérapie, une autre obligation fixée par l'ASN [7] prévoit la mise en place d'un système de management de la qualité, d'un système de déclaration interne des dysfonctionnements repérés et d'une étude systématique des risques encourus par les patients. Parallèlement à cette nouvelle règlementation, ces dernières années ont aussi vu se développer l'étude de la culture de sécurité des établissements. La culture de sécurité est un concept intégrateur qui englobe les comportements sûrs, l'esprit de sécurité, le climat de sécurité, l'apprentissage et la mémoire organisationnelle, les savoir-faire de prudence et le management de la sûreté [8,9]. Pour être développée, la culture de sécurité nécessite d'atteindre à la fois une sécurité réglée - basée sur l'application des normes et des règles fixées par l'organisation, et une sécurité gérée - fondée principalement sur les compétences et les réponses appropriées du collectif de travail [10]. Les travaux réalisés sur ce sujet par Nascimento [8,9] ont par exemple montré que les manipulatrices de radiothérapie sont régulièrement confrontées à des conflits de buts, les amenant à arbitrer entre plusieurs possibilités d'actions, toutes associées à la réalisation potentielle d'un risque. Par exemple, face à l'impossibilité de réaliser certaines opérations de 
contrôle pourtant obligatoires avant une séance de traitement, les manipulatrices doivent procéder à un arbitrage entre reporter ou ne pas reporter la séance prévue, décision qui dépend de la phase de traitement dans laquelle se trouve le patient. Pour des patients en début de traitement, elles décident généralement de reporter la séance, considérant ne pas être certaines de la dose correcte à délivrer à l'endroit prescrit ; pour des patients qui ont commencé leur traitement depuis un certain temps, elles décident de ne pas reporter la séance, considérant que le risque de compromettre la réussite du traitement en cas de report de la séance est plus important que celui d'administrer une dose incorrecte au patient. Dans le même esprit, face à un dossier non encore validé par le radiothérapeute, les manipulatrices doivent aussi procéder à un arbitrage du même type (reporter ou ne pas reporter la séance prévue), décision qui dépend cette fois de la connaissance qu'elles ont de leur collègue médecin, et en particulier de la façon dont il agit habituellement face aux cas simples ou encore des habitudes de collaboration de ce médecin avec ses collègues physiciens.

L'un des avantages de ce type d'études de la culture de sécurité au sein des établissements de soins est de mettre en lumière l'impact des conditions d'activité, et donc de l'organisation, sur les prises de décisions des professionnels en radiothérapie. Conjointement à cela, de tels travaux suggèrent aussi que ces professionnels doivent régulièrement procéder à des arbitrages entre plusieurs options, là où l'activité prescrite ne le prévoit pas. Il apparait que le choix de certaines options peut dépendre des relations entre professionnels (médecins radiothérapeutes, manipulateurs, physiciens médicaux et dosimétristes). Sur la base de ce résultat, et en sachant que la mise en œuvre de tout traitement par radiothérapie nécessite l'intervention et la collaboration de plusieurs professionnels à chacune des étapes du traitement, le premier objectif de notre recherche a été d'identifier toutes les situations dans lesquelles les prises de décision ne sont pas indépendantes des interactions sociales (que ce soit entre professionnels ou avec le patient) comme le prévoit pourtant l'organisation, mais 
sont en réalité dépendantes de telles interactions. Le deuxième objectif de notre recherche, concomitant au premier, a été d'analyser ces prises de décision au regard des risques qui y sont associés. D'un point de vue appliqué, notre recherche a pour visée la production collective et coordonnée de la sécurité des soins en radiothérapie, dont la mise en œuvre dépend de facteurs techniques, organisationnels, mais aussi psychosociaux [voir 11, 12, pour des recherches similaires].

\section{Méthode}

\section{Terrain de recherche et population}

Une analyse de l'activité a été menée au sein d'un établissement classé Centre de Lutte Contre le Cancer (CLCC), dans l'Est de la France. Sur le plan technique, le service de radiothérapie de cet établissement dispose des dernières avancées technologiques en radiothérapie. Son parc technique comprend six accélérateurs de particules ainsi qu'un scanner dosimétrique pour l'acquisition des données anatomiques des patients. D'un point de vue formel, la mise en œuvre d'un traitement par radiothérapie nécessite l'intervention et la collaboration de plusieurs professionnels. Après la pose de l'indication de radiothérapie pour le patient, le médecin radiothérapeute établit le plan de traitement approprié à sa pathologie et à son passé médical. Les manipulateurs réalisent ensuite un scanner dosimétrique afin d'acquérir les données anatomiques du patient et afin de permettre au médecin de définir les volumes à irradier. L'équipe de physique (physiciens médicaux et dosimétristes) procède alors à l'étude dosimétrique qui établit les propriétés techniques de la mise en œuvre du traitement sur l'accélérateur. Enfin, le traitement est délivré par un binôme de manipulateurs.

Le service de radiothérapie sollicité emploie au total 68 personnes, réparties en quatre unités de travail : (1) les médecins radiothérapeutes $(N=9),(2)$ les physiciens médicaux $(N=$ 6), les dosimétristes $(N=4)$, les ingénieurs de maintenance $(N=2)$, et l'assistante physicienne 
$(N=1)$ regroupés au sein de l'unité de physique, (3) les manipulateurs $(N=35)$, supervisés par la cadre de santé du service $(N=1)$, et (4) les assistantes médicales, secrétaires, et agents d'accueil $(N=10)$.

\section{Matériel et procédure}

Le cadre méthodologique général adopté ici a été celui proposé par Klein et collaborateurs car il a précédemment été appliqué avec succès à des problématiques de terrain complexes qui, comme la nôtre, nécessitent d'être abordées via différentes approches complémentaires [voir 13 pour une synthèse]. Dans cette logique, notre analyse a consisté en une découverte empirique de la situation de travail combinant trois procédés complémentaires de recueil des données utilisés de manière concomitante : l'analyse documentaire des protocoles et procédures formalisées, la conduite d'entretiens, et l'observation de l'activité.

Précisément, l'analyse documentaire a consisté à examiner les procédures qualité du service de radiothérapie, les protocoles d'utilisation des accélérateurs, les check-lists d'activité utilisées par les professionnels, ainsi que les supports de liaison et de transmission d'information. Cette analyse a permis d'appréhender la "tâche prescrite", c'est-à-dire la façon dont l'activité est formalisée par l'organisation du travail et doit se dérouler. Cette analyse documentaire a aussi permis de faire ressortir quels sont les professionnels concernés par la prise en charge des patients et à quelle phase de cette prise en charge ils doivent intervenir. C'est sur la base de ces informations que nous avons alors rencontré 32 professionnels ( 7 radiothérapeutes, 6 physiciens et dosimétristes, 1 ingénieur de maintenance, 10 manipulatrices, 1 cadre de santé, 6 secrétaires médicales et agents d'accueil, et 1 responsable du secrétariat). Ces rencontres ont permis d'approfondir notre familiarisation avec le terrain de recherche dans le sens où elles ont fait émerger la "tâche redéfinie" et fait découvrir l'activité réelle du service - c'est-à-dire la façon dont l'activité se déroule au quotidien aux dires et aux 
faits des professionnels. Elles ont aussi permis de développer un climat de confiance, engageant ainsi les participants dans la recherche et facilitant les échanges avec ces derniers dans le cadre de leur activité réelle. Lors de ces rencontres, des entretiens de type compréhensif $[14,15]$ d'une durée moyenne d'environ une heure ont été menés, soit au même moment que l'observation de l'activité, soit immédiatement après si le professionnel était avec un patient. Dans le détail, chaque entretien commençait par laisser le professionnel rencontré s'exprimer librement sur son activité professionnelle, alors même qu'il réalisait les gestes professionnels. Quand la personne abordait spontanément des questions d'arbitrage sur des décisions à prendre, il lui était demandé de préciser ces points. L'analyse préalable des documents permettait également de relancer la discussion sur des points susceptibles de représenter des nœuds décisionnels quand ceux-ci n'étaient pas spontanément abordés. Ces moments d'arbitrage étaient en effet essentiels à développer, puisque les séquences d'activités concernées par ces arbitrages ont ensuite constitué le focus de notre analyse de l'activité ${ }^{1}$. En d'autres mots, les précisions, relances, et autres questions que nous avons été amenés à poser concernaient essentiellement les décalages ou écarts entre la tâche prescrite d'un côté et la tâche redéfinie et l'activité réelle de l'autre côté, c'est-à-dire les moments où la procédure formelle prévue ne pouvait pas être respectée. Par exemple : que se passe-t-il quand le médecin n'a pas le dossier complet du patient au moment de prescrire son traitement ? Quelle pièce médicale est jugée incontournable pour démarrer le traitement ? Comment procéder quand le matériel requis pour le traitement n'est pas disponible ? Comment les physiciens modélisent la prescription médicale si elle ne permet pas de protéger parfaitement les organes

\footnotetext{
${ }^{1}$ Cette analyse de l'activité du service de radiothérapie a essentiellement porté sur la partie de la prise en charge qui va de l'adressage du patient jusqu'au démarrage des séances (identifiée comme la phase pré-thérapeutique et la préparation du traitement) et qui se situe donc en amont de la délivrance du traitement et du suivi posttraitement - car c'est là où les arbitrages ont majoritairement lieu et où le nombre de professionnels intervenant dans la prise en charge du patient est le plus important $[1,16]$.
} 
voisins de la zone à traiter ? Comment arbitrer entre confort du malade et précision de la zone à irradier ? Etc.

\section{Résultats}

Séquençage des étapes de la phase pré-thérapeutique et de la préparation du traitement L'analyse documentaire des protocoles et procédures formalisées a montré que la "tâche prescrite" du service de radiothérapie telle qu'elle est formalisée dans le manuel Qualité s'organise en 12 étapes, dont huit concernent la phase pré-thérapeutique et la préparation du traitement. Les entretiens avec les différents professionnels et l'observation de leur activité ont quant à eux permis de séquencer la phase pré-thérapeutique et l'activité de préparation du traitement en neuf étapes opérationnelles distinctes représentées dans la figure 1.

\section{Insérer figure $1 \mathrm{ici}$}

Ce qu'il est important de noter ici est que le séquençage effectif en neuf étapes ne recouvre que partiellement le découpage proposé dans le manuel Qualité. Certaines étapes mentionnées dans le manuel Qualité n'apparaissent pas du tout dans le discours des professionnels (par exemple, l'entretien paramédical à la phase pré-thérapeutique) alors que d'autres, non prévues dans le manuel, sont considérées par les professionnels comme des étapes d'importance (par exemple, la préparation du dossier dans cette même phase préthérapeutique). Le séquençage que nous proposons repose sur le discours et l'activité des professionnels que nous avons rencontrés et qui représentent les différents maillons du processus de prise en charge du patient. Il tient compte de ce que les professionnels ont évoqué comme relevant spécifiquement de leur activité mais aussi de ce qu'ils ont décrit comme relevant des interactions existantes avec les autres acteurs du service en amont et/ou 
en aval de leur intervention. Ce séquençage est présenté de façon détaillée dans la partie suivante. Les décalages avec le manuel Qualité y sont exposés.

Descriptif détaillé des étapes de la phase pré-thérapeutique et de préparation du traitement, des prises de décision, et des risques associés

Le traitement de la demande de prise en charge. Avant d'être transmise au service de radiothérapie, la demande de prise en charge a généralement été étudiée lors d'une Réunion de Concertation Pluridisciplinaire (RCP). Ces réunions, organisées pour chaque spécialité médicale hospitalière susceptible d'adresser des patients vers la radiothérapie, regroupent a minima un médecin spécialiste du domaine, ainsi qu'un médecin oncologue et un radiothérapeute. Ces professionnels décident ensemble de poser l'indication de radiothérapie pour le dossier étudié. Les demandes de prise en charge sont pour la plupart traitées par la secrétaire médicale (ou par le radiothérapeute lui-même quand ce dernier est directement sollicité par un confrère). Celle-ci renseigne une fiche de liaison avec les informations individuelles relatives au patient. Après s'être assurée qu'une indication de radiothérapie est ou va être posée, la secrétaire demande au service de provenance du patient que son dossier médical lui soit transmis dans les meilleurs délais. Enfin, elle planifie la première consultation du patient avec le radiothérapeute référent de cette prise en charge. Cette planification est effectuée selon deux critères : la spécialité de chacun des radiothérapeutes du service, et sa disponibilité pour une nouvelle consultation. A cette étape, ni prise de décision particulière ni risque associé majeur n'ont été repérés.

La préparation du dossier du patient. Le secrétariat a pour objectif de réunir l'ensemble des documents relatifs à l'état de santé du patient (dossier médical, résultats d'examens, ...), et de les mettre à disposition du radiothérapeute pour le jour de la consultation. Ces documents sont généralement détenus par le service hospitalier de 
provenance du patient. Malgré la consigne donnée par la secrétaire à son interlocuteur lors du traitement de la demande, il n'est pas rare qu'elle doive procéder à plusieurs relances afin d'obtenir ces documents. Ni prise de décision particulière ni risque associé majeur n'ont pu être repérés à cette étape. Cette étape apparait dans notre analyse parce que nous avons rencontré les secrétaires, considérées dans le service comme élément pertinent du processus de prise en charge des patients. Elles ont en effet un rôle très actif pour la reconstitution du dossier médical bien que cette étape n'apparaisse pas dans la cartographie du manuel Qualité car, en théorie, le dossier du patient est complet au moment où l'indication de radiothérapie est proposée.

La réalisation de la première consultation. Le médecin radiothérapeute découvre généralement le dossier du patient au moment de la consultation, sauf s'il a lui-même assisté à la RCP au cours de laquelle ce dossier a été présenté. Certains médecins préfèrent néanmoins prendre connaissance des dossiers de leurs nouveaux patients en amont de cette consultation, et demandent aux secrétaires que ces dossiers soient mis à leur disposition plus tôt. Après analyse du dossier médical du patient, le radiothérapeute procède à l'examen clinique. Ces deux étapes vont lui permettre d'établir son diagnostic, c'est-à-dire de valider l'indication de radiothérapie et de définir précisément les propriétés techniques du traitement : dose totale d'irradiation des cellules cancéreuses, fractionnement et durée du traitement, et procédé technique à utiliser. Le médecin définit également le délai de démarrage du traitement souhaité, et, le cas échéant, les examens d'imagerie complémentaires à réaliser afin de préparer la mise en œuvre de ce traitement. Lors de cette consultation, deux décisions importantes, à prendre par le radiothérapeute, ont été identifiées. La première décision concerne les propriétés du traitement à délivrer. Cette décision est essentiellement basée sur les informations à disposition du radiothérapeute et sur ses connaissances médicales. Le risque ici est de ne pas réussir à définir "la bonne dose, au bon endroit, au bon moment". La 
deuxième décision concerne la conduite à adopter en cas de manque de documents (dossier médical absent ou incomplet, résultats cliniques partiels, ...). Cette décision se prend en fonction de l'importance des informations manquantes. Dans le cas où trop d'éléments médicaux importants manquent, la première consultation peut être re-planifiée. Ce report de consultation comporte à la fois le risque de (trop) retarder le traitement et en même temps, la difficulté de l'annoncer au patient; il semble d'ailleurs que la question de qui fait cette annonce au patient ne soit pas clairement tranché : secrétaire médicale ou radiothérapeute ? On voit ici apparaitre à nouveau le rôle des secrétaires, qui semble avoir été négligé dans le manuel Qualité. Dans le cas où les éléments médicaux paraissent suffisants, il y a établissement d'un diagnostic qui demandera à être confirmé quand les pièces manquantes seront récupérées. Même si le diagnostic est posé, le risque est qu'il ne ne soit pas le plus approprié. Cependant, six des sept médecins participant à cette recherche indiquent préférer procéder à l'établissement d'un diagnostic provisoire, à confirmer par la suite, lorsqu'ils estiment cette solution possible.

La programmation du traitement. Elle se fait en fonction de deux critères, la date de démarrage du traitement souhaitée par le médecin et la disponibilité du ou des accélérateur(s) correspondant(s). La réunion de ces deux critères peut être difficile, principalement du fait de l'indisponibilité de l'accélérateur disposant de la technique choisie. Dans certains cas où le traitement peut être réalisé au moyen de techniques différentes (3D-conformationnelle ou IMRT, par exemple), le radiothérapeute doit alors choisir entre deux options : réaliser à la date de démarrage souhaitée le traitement au moyen d'une autre technique - avec le risque que ce traitement soit moins efficace, ou programmer à une date ultérieure le traitement du patient en fonction de la disponibilité de la machine - avec le risque que cette date soit (trop) tardive. Le choix de la solution se fait sur la base des éléments à disposition du 
radiothérapeute, de ses connaissances et de son expérience. La programmation du traitement et les difficultés éventuelles liées à cette étape n'apparaissent pas dans le manuel Qualité.

En revanche, les quatre étapes suivantes, qui relèvent de gestes très spécialisés avant la mise en route du traitement effectif, sont formalisées de la même façon dans l'activité prescrite par le manuel Qualité et dans l'activité réelle.

La réalisation du scanner dosimétrique (et l'acquisition de l'imagerie). Le scanner dosimétrique (seul ou couplé à d'autres examens d'imagerie comme l'IRM ou le PET scan) est nécessaire à la préparation de tout traitement de radiothérapie car il permet d'acquérir des données anatomiques utiles au calcul du plan de traitement du patient. Les résultats du scanner dosimétrique (et des éventuels examens complémentaires réalisés) sont ensuite récupérés par un physicien médical qui les charge sur l'espace de stockage numérique du service, afin de les rendre consultables par l'ensemble des professionnels du service. La réalisation du scanner est conditionnée par la technique de traitement choisie car la position à adopter par le patient lors de la réalisation du scanner doit être strictement identique à celle à adopter au cours des séances de rayons. Cette position est définie par le radiothérapeute lors de la première consultation. Dans cette situation, un problème fréquemment constaté par les deux manipulatrices en charge du scanner est l'incapacité du patient (notamment physique) à maintenir la position durablement dans le temps. Ce problème appelle une décision de la part du médecin référent du patient ou du médecin d'astreinte, en concertation avec les manipulatrices qui ont rapporté le problème. Soit la position décidée initialement est confirmée malgré les difficultés à la maintenir, avec le risque de mal irradier le patient. Soit la position est modifiée en tenant compte des recommandations avancées par les manipulatrices avec le risque de diminuer la qualité du traitement délivré du fait d'une position non "idéale".

La délinéation et la prescription. L'étude des résultats des examens d'imagerie permet au médecin d'affiner le diagnostic établi au cours de la première consultation. Il 
procède alors à la délinéation, étape durant laquelle il contoure sur les coupes d'imagerie les "volumes cibles", à irradier, et les "organes à risque", à protéger. Il établit ensuite la prescription du traitement à réaliser, puis la transmet avec les images contourées à l'unité de physique. De la même manière que lors de la première consultation, le radiothérapeute décide ici des propriétés médicales du traitement à mettre en œuvre, sur la base des éléments à sa disposition, et de son expertise médicale et technique. Ici aussi, le risque associé à cette décision est de ne pas réussir à définir "la bonne dose, au bon endroit, au bon moment".

Le calcul du plan de traitement. Il est réalisé par un physicien médical ou un dosimétriste. La répartition des dossiers à traiter entre les membres de l'unité de physique s'effectue en fonction de leur charge de travail et selon leur maîtrise de la technique de calcul à utiliser. Ce calcul vise à permettre l'irradiation des volumes cibles avec la dose définie par le radiothérapeute. Il s'agit d'assurer l'efficacité du traitement, tout en ne dépassant pas les seuils d'irradiation acceptables pour les organes à risques qui se trouvent à proximité. Il est toutefois fréquent que la complexité du cas traité empêche le physicien d'atteindre cette dose idéale. Tout en essayant de respecter les critères médicaux définis par le radiothérapeute, il doit alors décider sur la base de son expertise des propriétés techniques du traitement permettant d'arriver au meilleur plan de traitement possible : être au plus proche de la dose d'irradiation souhaitée pour les volumes cibles et au plus bas de la dose d'irradiation à laquelle les organes avoisinants sont soumis. Le risque associé à cette décision est de ne pas arriver au meilleur plan de traitement possible et donc mettre en œuvre un traitement de moindre efficacité, ou de plus grande dangerosité, que celui souhaité par le médecin.

\section{La validation du plan de traitement (et le chargement des données techniques sur}

l'accélérateur). La validation du plan de traitement est réalisée conjointement par le radiothérapeute référent du patient et le physicien médical qui a réalisé le calcul (ou à défaut un autre physicien), même si la décision finale revient au radiothérapeute, responsable de la 
prise en charge médicale du patient. Après la validation du plan de traitement, le physicien saisit les informations correspondantes sur le système informatique de l'accélérateur qui sera utilisé pour la délivrance des séances.

Valider conjointement les plans de traitement est une pratique instaurée dans le but de garantir la meilleure qualité de prise en charge possible pour le patient, et réduire les risques d'erreurs dans le plan de traitement. Cette étape est en effet l'occasion de débattre des résultats obtenus par le physicien, d'approfondir certains aspects du dossier, et de partager des connaissances techniques. Cette concertation aboutit fréquemment à un certain nombre de modifications dans le plan de traitement initial ; elle peut aussi être le lieu d'une confrontation entre critères médicaux et techniques et d'une vérification de l'adéquation entre ces deux aspects. Dans tous les cas, la qualité de la collaboration et plus globalement des relations entre le médecin et le physicien est primordiale dans cette étape de validation. Une mauvaise relation entre les deux professionnels peut les amener à décider de repousser cette concertation et donc le moment de validation du traitement ; il peut même arriver que le médecin et le physicien refusent cette concertation. Cette difficulté est évoquée unanimement par les physiciens et dosimétristes qui constituent un groupe professionnel soudé et qui défendent une compétence technique pouvant parfois se heurter aux choix médicaux. Il s'en suit le risque de retarder la mise en traitement du patient, ou celui de délivrer un traitement de moindre qualité avec une probabilité de ne pas détecter une erreur dans le plan de traitement établi.

La vérification du dossier du patient. Cette ultime étape avant la mise en traitement du patient consiste, pour les manipulatrices, à vérifier le contenu du dossier du patient ainsi que son adéquation avec la programmation technique de l'accélérateur. Cette phase qui apparait importante pour les professionnels n'est pas isolée en tant que telle dans le manuel Qualité. Afin de pouvoir réaliser ce contrôle dans de bonnes conditions, le protocole prévoit 
néanmoins que les manipulatrices disposent du dossier du patient au plus tard la veille de la mise en traitement. Malgré l’importance soulignée du respect des délais fixés pour la préparation des dossiers, il arrive régulièrement que les manipulatrices ne disposent pas à temps du dossier à vérifier. Dans cette situation, les manipulatrices doivent procéder à un arbitrage entre deux options : produire la santé des patients ou produire la sécurité des patients. Dans le premier cas, il s'agit pour les manipulatrices d'"accepter" le retard du dossier aussi longtemps que possible afin d'assurer la séance prévue pour le patient. Le risque associé à cette option est de ne plus disposer du temps nécessaire pour bien vérifier le dossier enfin arrivé et donc de ne pas détecter une erreur dans la programmation des rayonnements. Dans le second cas, il s'agit de décider que le retard du dossier ne leur permet plus d'assurer la mise en traitement dans de bonnes conditions et que celle-ci doit être reportée d'un jour. Le risque est alors de ne pas traiter le patient dans le délai imparti et de devoir lui annoncer directement cette nouvelle. Cette annonce peut être parfois faite le jour-même, alors que le patient est déjà présent en salle d'attente.

\section{Synthèse des prises de décisions identifiées et des risques associés}

En résumé, à l'exception des deux premières étapes (traitement de la demande et préparation du dossier), les sept autres étapes opérationnelles identifiées par les professionnels, représentent des moments clés de la prise en charge des patients, dans lesquelles une ou deux décisions doivent être prises, sans que le protocole formel n'offre de solutions faciles à ces questions. Ces décisions sont à assumer seul(e) ou en concertation avec d'autres professionnels, et peuvent concerner le traitement, le patient, ou les deux. Le tableau 1 fait la synthèse de ces prises de décisions, des professionnels impliqués dans celles-ci, et des risques potentiels.

Insérer tableau 1 ici 
Huit nœuds décisionnels ont été identifiés à travers cette recherche. Parmi ceux-ci, quatre reposent essentiellement sur les connaissances techniques et l'expérience du décideur et n'impliquent donc pas d'interactions sociales particulières (les décisions numérotées 1, 3, 5, et 6). En revanche, les quatre autres décisions identifiées (respectivement numérotées 2, 4, 7, et 8) nécessitent des interactions sociales entre professionnels ou entre professionnels et patient.

Concernant les interactions entre professionnels, celles-ci nécessitent de pouvoir échanger de façon constructive entre professionnels aux compétences variées. Ces échanges apparaissent parfois difficiles à mener, comme au moment de la validation du plan de traitement pendant lequel les échanges entre physiciens et médecins sont quelquefois vécus comme une confrontation entre deux logiques inconciliables. Plus généralement, de telles interactions sont d'autant plus mal vécues qu'elles interviennent dans un climat de méfiance vis-à-vis des positions de chaque partenaire impliqué et de défiance par rapport à la légitimité des décisions prises. Concernant les interactions entre les professionnels et les patients, les difficultés sont d'une autre nature puisque les échanges sont beaucoup plus asymétriques que précédemment. Lors de ces échanges où il s'agit généralement d'annoncer une mauvaise nouvelle au patient - comme par exemple le retard de la mise en place du traitement l'inconfort généré par la situation peut conduire à vouloir éviter l'interaction (en choisissant par exemple de mettre en place un traitement moins efficace ou d'administrer une dose d'irradiation sans vérification préalable du dossier, plutôt que de faire une telle annonce) ou à la déplacer vers d'autres collègues (les secrétaires, les manipulateurs).

\section{Discussion}

Notre recherche a visé à appréhender la manière dont certains facteurs psychosociaux interagissent avec les facteurs techniques et organisationnels dans le processus de prise de 
décisions dans un service de radiothérapie [12]. Conjointement, nous avons aussi tenté de comprendre en quoi certaines décisions peuvent générer des risques pour la santé ou le bienêtre des patients. De façon plus précise, il s'est agi d'identifier un certain nombre de situations dans lesquelles les prises de décisions sont directement liées aux interactions sociales entre professionnels ou avec le patient, puis d'analyser ces prises de décisions au regard des risques qui y sont associés. Pour ce faire, nous avons choisi de découvrir l'activité de radiothérapie à travers l'analyse documentaire des protocoles et procédures formalisées et les rencontres avec les professionnels concernés. Ces rencontres - sous forme d'entretiens de type compréhensif et d'observation de l'activité - ont mis en évidence que les professionnels séquencent leur activité en neuf étapes importantes, allant de la réception du dossier du patient jusqu'à la mise en place de son traitement. Au sein de plusieurs de ces étapes, nous avons repéré que la moitié des décisions importantes à prendre sont dépendantes des relations sociales au sein du service. Bien entendu, ce type de décisions est difficilement formalisable dans une procédure standard dans la mesure où les relations sociales dépendent étroitement des partenaires impliqués. Cela signifie que la prise de risque en radiothérapie dépasse la maitrise de l'organisation de l'activité et du geste technique pour inclure une dimension plus individuelle. Ce résultat n'a rien d'étonnant puisque la prise en charge oncologique requiert précisément l'intervention coordonnée d'un ensemble d'acteurs [11, 17]. Par ailleurs, toutes ces décisions sont délicates à prendre dans la mesure où elles impliquent directement un surcroit de risques potentiels pour la santé du patient : il peut s'agir de la décision d'annoncer à un patient qu'on retarde la mise en place de son traitement soit parce que le dossier incomplet ne permet pas au médecin de poser une indication de traitement, soit parce que les manipulatrices ne peuvent pas vérifier les éléments prouvant l'adéquation entre le calcul du traitement et le rayonnement programmé. Il peut s'agir aussi de décider pour le médecin, en concertation avec les manipulatrices, de changer la position dans laquelle le patient doit recevoir son traitement 
pour privilégier son confort, ou au contraire de lui expliquer que malgré les douleurs et l'inconfort, il s'agit de recevoir le traitement de la manière la plus efficace possible. D'un point de vue psychosocial, la difficulté associée à ces décisions réside dans le fait d'affronter le patient déjà fragilisé par les implications de sa maladie, pour lui annoncer des nouvelles généralement désagréables. Ces interactions sont vectrices d'émotions négatives pour le patient comme, sans doute, pour le thérapeute lui-même, même si la nature des réactions reste différente. Parallèlement, cette recherche a mis en évidence que cette question des relations sociales et de leur effet sur les prises de décisions touche aussi les professionnels entre eux. Nous avons ainsi repéré la difficulté que pouvait générer l'obligation de valider le calcul du traitement de façon conjointe entre radiothérapeutes et physiciens. Dans un certain nombre de cas, ces professionnels retardent l'échéance de cette confrontation, voire la refusent, repoussant d'autant la date de mise en traitement du patient et/ou augmentant la probabilité de ne pas détecter une erreur. Parmi les raisons à l'origine de telles décisions, il y a vraisemblablement le manque de confiance mutuelle ou bien la difficulté à considérer ce partenariat comme utile et efficient.

A ce niveau, l'intérêt de notre démarche - qui vise in fine à améliorer la sécurité des soins en radiothérapie - est de poursuivre l'analyse afin de comprendre ces principaux "nœuds décisionnels" qui reposent sur la coopération entre partenaires tenus d'arbitrer entre différentes options afin de prendre une décision. Dans cette optique, il va s'agir de confronter les observations faites à travers notre analyse préliminaire aux processus psychosociaux sousjacents aux décisions prises.

Concernant la relation médecin-patient, des recherches en psychologie sociale ont montré que le comportement du médecin envers son patient est impacté par des variables affectives. Par exemple, des entretiens conduits auprès de médecins oncologues ont mis en avant que le fait de devoir annoncer une mauvaise nouvelle à un patient, lors de rencontres en 
face à face, génère chez ces médecins des manifestations émotionnelles négatives telles que l'anxiété, la difficulté ou le malaise [18]. D'autres travaux ont mis expérimentalement en évidence que la proximité physique du personnel soignant avec les malades modifie leurs réactions émotionnelles face à ces personnes : des étudiants infirmiers imaginant apporter un réconfort personnel ou un soin courant expriment plus de pitié, d'impuissance et de gêne face à une personne souffrant d'une maladie potentiellement létale que face à une personne hospitalisée pour une maladie moins grave $[19,20]$. En retour, ces réactions émotionnelles ont des conséquences sur la façon dont les professionnels soutiennent leurs patients, en générant par exemple des comportements de mise à distance du malade et de focalisation sur les aspects techniques de la pathologie [19,21]. Tous ces résultats appuient l'idée que les interactions entre professionnels et patients génèrent des émotions et que celles-ci peuvent influencer certaines prises de décisions à annoncer directement aux patients. A ce niveau, l'une des pistes de travail à suivre est donc de mesurer plus directement les réactions affectives générées par les interactions rencontrées lors de la préparation du traitement en oncologie, et d'analyser comment ces réactions impactent les décisions prises (et notamment l'annonce au patient) et les risques associées à celles-ci.

Concernant les relations entre professionnels, le champ de la psychologie des organisations s'est beaucoup intéressé à la compréhension des relations entre collègues sur le lieu de travail. L'un des résultats des nombreux travaux menés en ce sens a montré que le principal facteur explicatif de la qualité des relations entre collègues est la confiance que l'individu accorde à ses collègues [22,23], confiance souvent associée à la notion de compétence perçue [24, 25]. La modélisation de la confiance proposée par McAllister [26] va aussi dans ce sens. Selon cet auteur, le concept de confiance comporte deux facettes : une facette cognitive basée sur les compétences et la fiabilité perçue chez les collègues, et une facette affective reflétant une attention mutuelle entre collègues et reposant en particulier sur 
la fréquence et la qualité des interactions entre les collègues. Malheureusement, ce modèle théorique n'a reçu que peu de preuves empiriques. Dans le secteur de la santé, et notamment en oncologie, les travaux ont plutôt porté sur la relation de confiance qui lie le patient à son médecin [27, 28]. Dans les quelques travaux qui ont abordé la notion de confiance entre collègues, il a principalement été question de la confiance du professionnel envers son environnement de travail au sens large, intégrant ainsi la relation aux collègues de travail mais seulement comme un épiphénomène de l'environnement de travail [29]. A l'avenir, cet axe de recherche nous semble cependant intéressant à approfondir. Les résultats obtenus dans notre recherche nous laissent en effet penser que le niveau de confiance entre collègues peut directement influencer les prises de décisions réalisées en situation d'interaction avec un ou plusieurs collègue(s). L'étude de cette dimension pourrait donc permettre de mieux comprendre ces prises de décisions, et à terme, améliorer la qualité de ces décisions et plus globalement la qualité des soins.

En conclusion, notre recherche a privilégié la confrontation directe avec le terrain, afin d'identifier les pratiques réelles de travail et de proposer aux professionnels concernés des conclusions spécifiques à leurs situations de travail. Ces conclusions, propres à l'organisation du service de radiothérapie qui nous a accueillis pour cette recherche, doivent maintenant trouver une validité écologique grâce à l'étude plus spécifique des processus à l'œuvre dans les prises de décisions issues des interactions entre professionnels ou avec les patients. C'est avec cette ambition que les prochaines investigations devront se poursuivre. 


\section{Références bibliographiques}

1. Chauvet B, Mahé MA, Maingon P, et al (2013) Livre blanc de la radiothérapie en France 2013. Douze objectifs pour améliorer un des traitements majeurs du cancer. [White paper on radiation oncology in France. Twelve proposals to improve a major cancer treatment]. Cancer Radiother 17:2-72

2. Mazeron R, Aguini N, Deutsch E (2013) Analyse des risques en radiothérapie : état des lieux. [Risk analysis in radiation therapy: State of the art]. Cancer Radiother 17:308316

3. Gerard JP, Thariat J, Giraud P, et al (2010) Histoire de l'évolution des techniques de radiothérapie. [Past, present and near future of techniques in radiation oncology]. B Cancer 97:743-751

4. Autorité de Sûreté Nucléaire - Inspection Générale des Affaires Sociales (2007) Résumé du rapport ASN n² 2006 ENSTR 019 - IGAS n RM 2007- 015P sur l'accident de radiothérapie d'Epinal. Retrieved from http://www.ladocumentationfrancaise.fr/rapports-publics/074000198

5. Peiffert D, Simon JM, Eschwege F (2007) L'accident d'Epinal : passé, présent, avenir. [Epinal radiotherapy accident: past, present, future]. Cancer Radiother 11: 309-312

6. Haute Autorité de Santé (2011) La culture de sécurité des soins : du concept à la pratique. Retrieved from http://www.has-sante.fr/portail/jcms/c_1239410/fr/mettre-en-oeuvrela-gestion-des-risques-associes-aux-soins-en-etablissement-de-sante

7. Autorité de Sûreté Nucléaire (2008) Décision n²008-DC-103 du 1er juillet 2008 fixant les obligations d'assurance qualité en radiothérapie. Retrieved from http://www.asn.fr/Media/Files/00-Bulletin-officiel/2008_DC_103.pdf

8. Nascimento A (2011) Sécurité des patients et culture de sécurité : une revue de la littérature. Ciência \& Saúde Coletiva 16:3591-3602 
9. Nascimento A, Falzon P (2009) Produire la santé, produite la sécurité : récupérations et compromis dans le risque des manipulatrices en radiothérapie. Activités 6:3-23

10. Daniellou F, Boissières I, Simard M (2010) Les facteurs humains et organisationnels de la sécurité industrielle : un état de l'art. Les Cahiers de la Sécurité Industrielle 2010-02

11. Munoz MI, Barcellini F, Mollo V, et al (2015) Coopération asynchrone en milieu médical : prise en compte de la gestion de la variabilité liée au patient dans la conception d'un outil de workflow. Activités 12:70-88

12. Nascimento A, Falzon P (2012) Producing effective treatment, enhancing safety: Medical physicists' strategies to ensure quality in radiotherapy. Appl Ergon 43: 777-789

13. Klein JT, Grossenbacher-Mansuy W, Haberli R, et al (2001) Transdisciplinarity: Joint problem solving among science, technology, and society - an effective way for managing complexity. Birkhäuser, Basel

14. Glaser BG, Strauss AL (2010) La découverte de la théorie ancrée : stratégies pour la recherche qualitative. Armand Colin, Paris

15. Kaufmann JC (1996) L'entretien compréhensif. Nathan, Paris

16. Société Française de Radiothérapie Oncologique (2007) Guide des procédures de radiothérapie externe 2007 . Retrieved from http://www.hassante.fr/portail/upload/docs/application/pdf/2008/guide_de_rth_des_tu meurs_v7_complet.pdf

17. Motté F (2012) Le collectif transverse : un nouveau concept pour transformer l'activité. In: Beguin P, Puyeo V (eds) Actes du Congrès de la Self. Lyon, France

18. Desauw A, Christophe V, Antoine P, et al (2009) Quelle perception les praticiens ont-ils de l'annonce de mauvaises nouvelles en oncologie ? Analyse qualitative du vécu et des stratégies de régulation émotionnelle. Psycho-oncologie 3:134-139 
19. Dijker AJ, Raeijmaekers F (1999) The influence of seriousness and contagiousness of disease on emotional reactions to ill persons. Psychol Health 14:131-141

20. Peter E, Liaschenko J (2004) Perils of proximity: A spatiotemporal analysis of moral distress and moral ambiguity. Nurs Inq 11:218-225

21. Christophe V, Antoine P, Leroy T, et al (2009) Évaluation de deux stratégies de régulation émotionnelle : la suppression expressive et la réévaluation cognitive. Eur Rev Appl Psychol 59:59-67

22. Holton JA (2001) Building trust and collaboration in a virtual team. Team Performance Management 7:36-47

23. Lucas LM (2005). The impact of trust and reputation on the transfer of best practices. Journal of Knowledge Management 9:87-101

24. Butler JK (1991) Toward understanding and measuring conditions of trust: Evolution of a conditions of trust inventory. J Manage 17:643-663

25. Cook J, Wall T (1980) New work attitude measures of trust, organizational commitment and personal need non-fulfilment. J Occup Psychol 53:39-52

26. McAllister DJ (1995) Affect- and cognition-based trust as foundations for interpersonal cooperation in organizations. Acad Manage J 38:24-59

27. Gupta S, Brenner AT, Ratanawongsa N, et al (2014) Patient Trust in Physician Influences Colorectal Cancer Screening in Low-Income Patients. Am J Prev Med 47:417-423

28. Smets E, Hillen MA, Douma KF, et al (2013) Does being informed and feeling informed affect patients' trust in their radiation oncologist? Patient Educ Couns 90:330-337

29. Gilson L, Palmer N, Schneider H (2005) Trust and health worker performance: Exploring a conceptual framework using South African evidence. Soc Sci Med 61:1418-1429 
Figure 1 Séquençage de la phase pré-thérapeutique et de la préparation du traitement

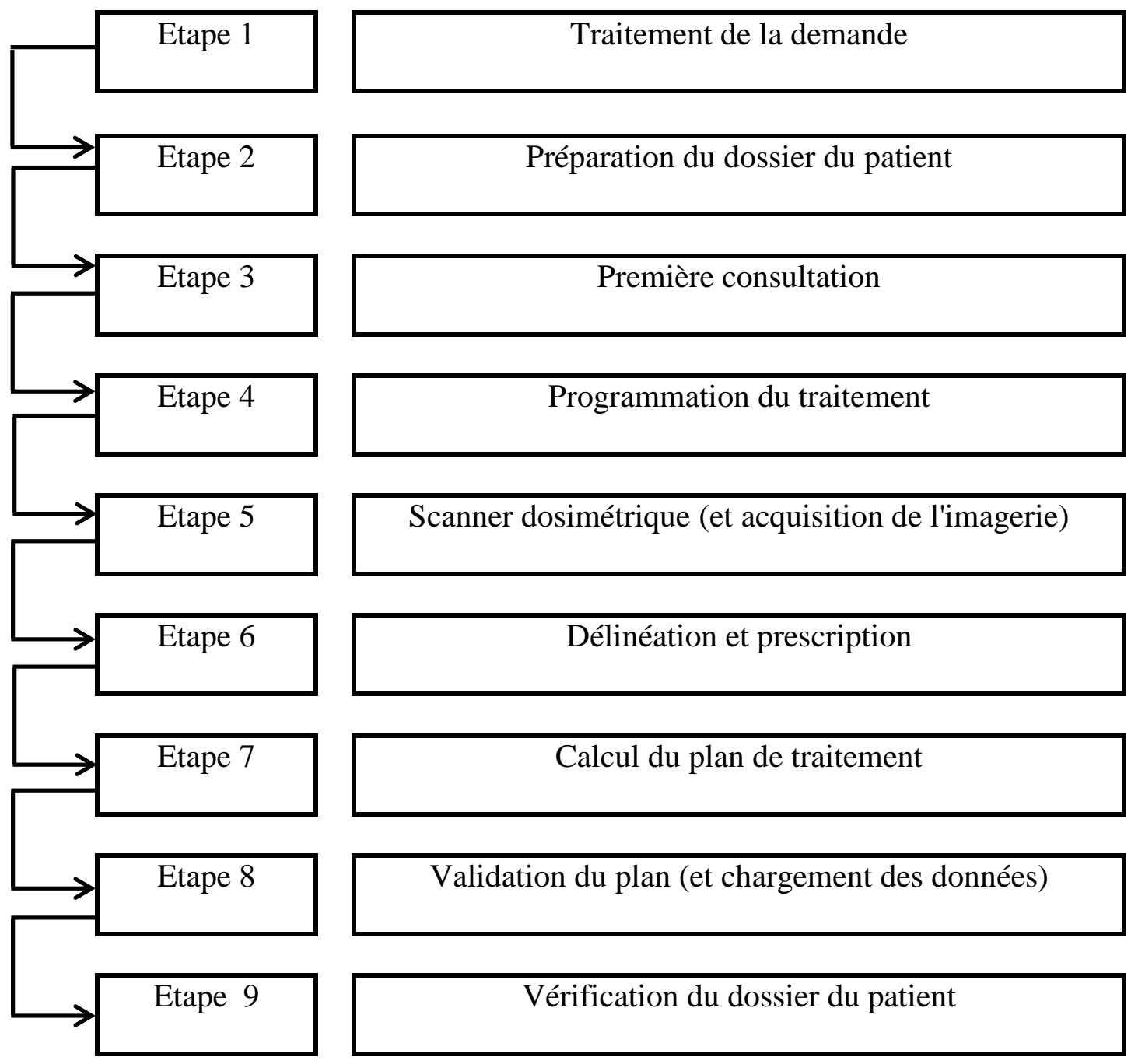


Tableau 1 Synthèse des prises de décisions identifiées (avec les professionnels concernés) et des risques qui y sont associés pour chaque étape d'activité

\begin{tabular}{|c|c|c|}
\hline Etape d'activité & $\begin{array}{c}\text { Décisions prises } \\
\text { (professionnels concernés) }\end{array}$ & Risques associés à la décision \\
\hline \multicolumn{3}{|l|}{$\begin{array}{l}1: \text { Traitement de la } \\
\text { demande }\end{array}$} \\
\hline \multicolumn{3}{|l|}{$\begin{array}{l}2: \text { Préparation du } \\
\text { dossier }\end{array}$} \\
\hline \multirow[t]{2}{*}{$\begin{array}{l}3 \text { : Première } \\
\text { consultation }\end{array}$} & $\begin{array}{l}\text { Décision } \mathrm{n}^{\circ} 1 \text { : traitement à délivrer } \\
\text { (radiothérapeute) }\end{array}$ & $\begin{array}{c}\text { Ne pas définir la "bonne dose", le "bon } \\
\text { endroit" ou le "bon moment" pour l'irradiation } \\
\text { du patient }\end{array}$ \\
\hline & $\begin{array}{l}\text { Décision } n^{\circ} 2 \text { : conduite à adopter en } \\
\text { cas de manque de documents } \\
\text { (radiothérapeute) }\end{array}$ & $\begin{array}{c}\text { Poser une décision médicale qui ne soit pas la } \\
\text { plus adéquate ou retarder le traitement du } \\
\text { patient et devoir lui annoncer }\end{array}$ \\
\hline $\begin{array}{l}4 \text { : Programmation } \\
\text { du traitement }\end{array}$ & $\begin{array}{l}\text { Décision } \mathrm{n}^{\circ} 3 \text { : techniques de } \\
\text { traitement à privilégier en fonction de } \\
\text { leur efficacité et de leur délai } \\
\text { (radiothérapeute) }\end{array}$ & $\begin{array}{l}\text { Réaliser un traitement de moindre efficacité } \\
\text { mais sans délai ou réaliser un traitement } \\
\text { efficace mais dont la date de démarrage } \\
\text { diminue ses chances de réussite }\end{array}$ \\
\hline $\begin{array}{l}5: \text { Scanner } \\
\text { dosimétrique (et } \\
\text { acquisition de } \\
\text { l'imagerie) }\end{array}$ & $\begin{array}{l}\text { Décision } \mathrm{n}^{\circ} 4 \text { : positionnement du } \\
\text { patient à modifier en fonction des } \\
\text { difficultés constatées suite au scanner } \\
\text { (radiothérapeute et manipulatrices) }\end{array}$ & $\begin{array}{l}\text { Mal irradier le patient (qui ne peut pas } \\
\text { respecter le positionnement décidé } \\
\text { initialement) ou procéder à un traitement } \\
\text { moins efficace que celui prévu initialement }\end{array}$ \\
\hline $\begin{array}{l}6 \text { : Délinéation et } \\
\text { prescription }\end{array}$ & $\begin{array}{l}\text { Décision n }{ }^{\circ} \text { : propriétés médicales } \\
\text { du traitement à valider et préciser } \\
\text { (radiothérapeute) }\end{array}$ & $\begin{array}{l}\text { Ne pas définir la "bonne dose", le "bon } \\
\text { endroit" ou le "bon moment" pour l'irradiation } \\
\text { du patient }\end{array}$ \\
\hline $\begin{array}{l}7 \text { : Calcul du plan de } \\
\text { traitement }\end{array}$ & $\begin{array}{l}\text { Décision } \mathrm{n}^{\circ} 6: \text { propriétés techniques } \\
\text { du traitement à rechercher puis } \\
\text { choisir (physicien ou dosimétriste) }\end{array}$ & $\begin{array}{l}\text { Ne pas arriver au meilleur plan de traitement } \\
\text { possible (mettre en œuvre un traitement de } \\
\text { moindre efficacité ou de plus grande } \\
\text { dangerosité) }\end{array}$ \\
\hline $\begin{array}{l}8: \text { Validation du } \\
\text { plan (et chargement } \\
\text { des données) }\end{array}$ & $\begin{array}{l}\text { Décision } \mathrm{n}^{\circ} 7 \text { : plan de traitement à } \\
\text { discuter et à valider sur le plan } \\
\text { médical (radiothérapeute et physicien } \\
\text { ou dosimétriste) }\end{array}$ & $\begin{array}{l}\text { Retarder la mise en traitement du patient (du } \\
\text { fait d'une augmentation du délai de validation } \\
\text { du plan) et/ou mettre en œuvre un traitement } \\
\text { de moindre qualité et/ou ne pas détecter une } \\
\text { erreur dans le plan (en cas de mauvaise } \\
\text { relation entre les deux professionnels) }\end{array}$ \\
\hline $\begin{array}{l}9 \text { : Vérification du } \\
\text { dossier du patient }\end{array}$ & $\begin{array}{l}\text { Décision } n^{\circ} 8: \text { conduite à adopter en } \\
\text { cas de retard du dossier au poste de } \\
\text { traitement (manipulatrices) }\end{array}$ & $\begin{array}{l}\text { Ne pas détecter une erreur dans la } \\
\text { programmation des rayonnements (pouvant } \\
\text { conduire à une mauvaise irradiation du patient) } \\
\text { ou repousser la mise en traitement et devoir } \\
\text { l'annoncer le jour-même au patient (qui se } \\
\text { trouve parfois déjà dans la salle d'attente) }\end{array}$ \\
\hline
\end{tabular}

